\title{
Ionizing energy depositions after fast neutron interactions in silicon
}

\author{
Benedikt Bergmann, Stanislav Pospisil, Ivan Caicedo, \\ James Kierstead, Helio Takai, and Erik Frojdh
}

Submitted to IEEE Transaction on Nuclear Science

June 2016

Physics Department

Brookhaven National Laboratory

\author{
U.S. Department of Energy \\ USDOE Office of Science (SC), \\ High Energy Physics (HEP) (SC-25)
}

\footnotetext{
Notice: This manuscript has been authored by employees of Brookhaven Science Associates, LLC under Contract No. DE- SC0012704 with the U.S. Department of Energy. The publisher by accepting the manuscript for publication acknowledges that the United States Government retains a non-exclusive, paid-up, irrevocable, world-wide license to publish or reproduce the published form of this manuscript, or allow others to do so, for United States Government purposes.
} 


\section{DISCLAIMER}

This report was prepared as an account of work sponsored by an agency of the United States Government. Neither the United States Government nor any agency thereof, nor any of their employees, nor any of their contractors, subcontractors, or their employees, makes any warranty, express or implied, or assumes any legal liability or responsibility for the accuracy, completeness, or any third party's use or the results of such use of any information, apparatus, product, or process disclosed, or represents that its use would not infringe privately owned rights. Reference herein to any specific commercial product, process, or service by trade name, trademark, manufacturer, or otherwise, does not necessarily constitute or imply its endorsement, recommendation, or favoring by the United States Government or any agency thereof or its contractors or subcontractors. The views and opinions of authors expressed herein do not necessarily state or reflect those of the United States Government or any agency thereof. 


\title{
Ionizing energy depositions after fast neutron interactions in silicon
}

\author{
Benedikt Bergmann, Stanislav Pospisil, Senior Member, IEEE, Ivan Caicedo, James Kierstead, Member, IEEE, \\ Helio Takai, Member, IEEE, and Erik Frojdh
}

\begin{abstract}
In this study we present the ionizing energy depositions in a $300 \mu \mathrm{m}$ thick silicon layer after fast neutron impact. With the Time-of-Flight (ToF) technique, the ionizing energy deposition spectra of recoil silicons and secondary charged particles were assigned to (quasi-) monoenergetic neutron energies in the range from $180 \mathrm{keV}$ to hundreds of $\mathrm{MeV}$. We show and interpret representative measured energy spectra. By separating the ionizing energy losses of the recoil silicon from energy depositions by products of nuclear reactions, the competition of ionizing (IEL) and non-ionizing energy losses (NIEL) of a recoil silicon within the silicon lattice was investigated. The data give supplementary information to the results of a previous measurement and are compared with different theoretical predictions.
\end{abstract}

Index Terms-Neutron radiation effects, Nonionizing energy loss, Semiconductor detectors

\section{INTRODUCTION}

$\mathbf{S}$ ILICON has become a ubiquitous material for the fabrication of active sensor layers in particle and radiation detectors. It is also the element of choice in the semiconductor industry for the manufacture of electronic devices. In several applications, silicon based devices need to operate while exposed to harsh radiation environments. For instance, in space, trapped particles in the Earth magnetosphere, cosmic rays and energetic particles from coronal mass ejection constantly bombard electronics located aboard satellites. In the terrestrial environment electronic devices and sensors are exposed to natural radiation produced by cosmic rays, e.g. avionics, or man-made radiation, such as encountered in hadron therapy facilities and nuclear reactors. One of the most challenging anthropogenic radiation field is the one found at collider accelerator facilities. Sensors used for particle tracking, e.g. silicon micro strip detectors, must perform well at the large hadron collider (LHC) when exposed to fluences up to $10^{14} \mathrm{~cm}^{-2}$ per operational LHC year [1]. As newer technologies permit the design and implementation of highly integrated sensors and electronics devices, the understanding of how hadrons interact with silicon at a microscopic level becomes of paramount importance. These interactions induce damage in the device determining their useful lifetime and performance.

B. Bergmann and S. Pospisil are with the Institute of Experimental and Applied Physics, Czech Technical University in Prague, Horska 3a/22, 128 00 Praha 2-Albertov, Czech Republic.

E-mail: benedikt.bergmann@utef.cvut.cz

J. Kierstead and H. Takai are with the Brookhaven National Laboratory, P.O. Box 5000, Upton, NY 11973-5000, United States.

E. Frojdh was with the CERN, CH-1211 Geneva 23, Switzerland.
There are two general classes of radiation damage to be considered: permanent and transient effects. Permanent damages are those that over time decrease the overall particle detector performance by decreasing the charge collection efficiency. This may happen due to an increase in the leakage current, leading to the need to increase the bias voltage required to fully deplete the sensor layer. Displacement of silicon atoms in the crystalline structure and the trapping of charge near the transistor gate insulator are the most common damages caused by radiation. A detailed discussion of how permanent damage degrades the detector performance can be found in [2]. Transient effects are best known in the literature as single event effects or soft errors. They are induced by a localized deposition of charge due to the passage of heavily ionizing particles near the electronic circuit sensitive volume. In the eventuality that this charge is larger than a critical charge it will force a change in the logic state of a cell. In space, high energy heavy ions are the ones responsible for inducing single event effects. In the terrestrial and accelerator environment the most important hadrons inducing soft errors are neutrons [3]. For neutrons, to impact high ionization densities they must produce a heavily ionizing charged particle, such as a recoiling silicon nucleus, or induce a nuclear reaction. Due to the statistical nature of nuclear interactions and scattering, the rate of single event effects is difficult to predict. Neutrons must produce the heavily ionizing ion near the device sensitive volume. In contrast, permanent damage is due to the accumulation of sites that are damaged or charged. Thus, the device lifetimes can usually be predicted given the knowledge of neutron flux and the maximum fluence for device operability.

In this work, we present a detailed study of the energy depositions caused by neutron interactions with silicon. The silicon layer of a Timepix 3 [4] device was used as a neutron target and exposed to the white energy spectrum neutron beam at the Los Alamos Neutron Science Center. The Timepix3 is a finely segmented detector that measures both, the deposited energy and time of arrival for each of the pixels struck by a particle. As the accelerator is a pulsed facility, a ToF measurement has been used to determine the neutron energy that induces the interaction. The latter is characterized by a pattern imprinted in the pixel array. Integrating the per pixel energy depositions of the secondary particles, we determined the ionizing energy depositions in a silicon layer, that are relevant for the single event effect modeling.

We also present a detailed study of the non ionizing energy 
loss (NIEL) and the ionizing energy loss (IEL) of silicon atoms after a neutron impact. Theoretical interest in this topic arose in 1963, when Lindhard et al. [5] presented integral equations to predict the NIEL and IEL. A previous measurement of the ionizing energy losses of a recoil silicon within a silicon lattice was carried out by Sattler [6] in 1965, who performed spectroscopic measurements with a silicon surface barrier detector in (quasi-)monoenergetic neutron beams (with energies in the range from $159 \mathrm{keV}$ to $23.6 \mathrm{MeV}$ ) at the Van-de-Graaff accelerator at the Los Alamos Scientific Laboratory.

Although progress has been made in the understanding of radiation damage and single event effects prediction through simulations (see for example [3]) there is shortage of experimentally measured data for model verification. The presented study uses a technologically sophisticated detector to provide data that can be a valuable input for further model development.

\section{THEORETICAL BACKGROUND}

For neutrons, the permanent damage in semiconductor devices is predominantly caused by the nuclear neutron scattering processes. Silicon recoil atoms, commonly referred to as primary knock-on atoms (PKA), are produced and traverse the sensor material. They lose energy by atomic processes (the displacement of atoms from their lattice positions) and electronic ionization. In particular, bulk damage (displacement damage), which is assumed to degrade the sensor layer properties, is the result of the NIEL.

For high PKA energies, an incoming particle can even produce clusters of defects. The PKA displaces atoms from their lattice position, which then again displace atoms from their lattice positions, resulting in a cascade-like propagation of atomic displacements. Kinchin and Pease derived a simple model to estimate the average number of stable defects, which are referred to as Frenkel pairs (FP) [7]. Their model was based on the assumptions of atomic hard-core scattering and a sharp threshold for permanent displacement $E_{\mathrm{d}}$. Moreover, crystal lattice effects were neglected. Using extensive computer simulations Norgett and Robinson further investigated this cascade model [8], [9]. Their studies resulted in the modified KinchinPease formula, which gives the average number of FP as a function of the damage energy $E_{\mathrm{de}}$ :

$$
\left\langle N_{\mathrm{FP}}\right\rangle= \begin{cases}1 & , \text { for } E_{\mathrm{d}}<E_{\mathrm{de}} \leq \frac{2 E_{\mathrm{d}}}{\zeta} \\ \zeta \frac{E_{\mathrm{de}}}{2 E_{\mathrm{d}}} & , \text { for } E_{\mathrm{de}}>\frac{2 E_{\mathrm{d}}}{\zeta}\end{cases}
$$

where $\zeta$ is the displacement efficiency and $E_{\mathrm{d}}$ the threshold energy for the permanent displacement of an atom from its lattice site [2], [7], [10].

In the case of neutron impact on silicon, the values $\zeta=0.8$ and $E_{\mathrm{d}}=25 \mathrm{eV}$ can be found in [2]. $E_{\mathrm{d}}$ is assumed to be isotropic and sharp. With this, the problem of the determination of stable defects is reduced to the knowledge of damage energy, i.e. the non-ionizing energy depositions.

Based on the integral equations by Lindhard et al. [5], the energy depositions $E_{\text {de }}$ can be described by the Norgett-
Torrens-Robinson expressions (see equations (6)-(9) in [8]). For a recoil silicon in a silicon lattice, they read as:

$$
E_{\mathrm{de}}=\frac{E_{\mathrm{Si}}}{1+k \times g(\epsilon)},
$$

with $k=0.1462, \epsilon=1.014 \times 10^{-2} \times Z_{\mathrm{Si}}^{-7 / 3} \times E_{\mathrm{Si}}=2.147 \times$ $10^{-5} E_{\mathrm{Si}}$ and the universal function

$$
g(\epsilon)=3.4008 \times \epsilon^{1 / 6}+0.40244 \times \epsilon^{3 / 4}+\epsilon \text { [8, [2], [1]. }
$$

The partitions of energy depositions are given by

$$
f_{\mathrm{NIEL}}=\frac{E_{\mathrm{de}}}{E_{\mathrm{Si}}}=\frac{1}{1+k \times g(\epsilon)}
$$

and

$$
f_{\text {ion }}=1-\frac{E_{\mathrm{de}}}{E_{\mathrm{Si}}}=\frac{k \times g}{1+k \times g(\epsilon)}
$$

where $f_{\text {ion }}$ is the quantity whose energy dependence was investigated in section IV-B of the presented work.

Akkerman and Barak [12] described a novel approach to calculate the ionizing energy losses, which was reported to better describe the partition function for low recoil energies. Their results can be expressed in terms of the Norgett-TorrensRobinson formalism by using the modified universal function (see also the discussion in [13]):

$$
g_{\mathrm{A}}(\epsilon)=0.90656 \times \epsilon^{1 / 6}+1.6812 \times \epsilon^{3 / 4}+0.74422 \times \epsilon .
$$

\section{DETECTOR SETUP AND DATA EVALUATION TECHNIQUE}

\section{A. Los Alamos Neutron Science Center}

The experiment was performed at the Target 4 Flight Path 30R of the Weapon Neutron Research facility in the Los Alamos Neutron Science Center [14]. Neutrons with a broad spectrum from hundreds of $\mathrm{keV}$ up to approximately $800 \mathrm{MeV}$ are produced by an $800 \mathrm{MeV}$ LINAC proton beam hitting a cylindrical tungsten target. In the spallation process, each proton creates 10-20 neutrons. The detector was situated at a distance of $21.3 \mathrm{~m}$ from the interaction point and irradiated perpendicularly. To allow users to perform ToF measurements, the proton beam is divided into macro-pulses with $625 \mu \mathrm{s}$ length, consisting of sharp micro-pulses (FWHM: $125 \mathrm{ps}$ ). The separation between two consecutive micro-pulses is around $1.8 \mu \mathrm{s}$, giving a wrap around energy of $645 \mathrm{keV}$ for the $20 \mathrm{~m}$ flight path. By using the last micro-pulse within the bunch of macro-pulses we determined and subtracted the contribution of neutrons with lower energies than the wrap around to the presented energy spectra and we extended the investigated neutron energy range down to $176 \mathrm{keV}$.

\section{B. Timepix3 detectors}

A Timepix 3 detector, equipped with a $300 \mu \mathrm{m}$ thick silicon sensor was used. It was developed in the framework of the Medipix3 collaboration [15] as the successor to the Timepix chip [16]. The data acquisition was performed with a FitPix controlled by the PiXet software. Both, the readout and the software were developed and produced by ADVACAM s.r.o 1

${ }^{1}$ ADVACAM s.r.o., Na Balkane 2075/70, 13000 Praha 3, Czech Republic. 
in collaboration with the Institute of Experimental and Applied Physics, Czech Technical University in Prague.

The Timepix3 readout chip, designed in $130 \mathrm{~nm}$ CMOS technology, divides the active sensor into a matrix of $256 \times 256$ pixels with $55 \mu \mathrm{m}$ pixel to pixel distance. The chip design allows a simultaneous measurement of the time and the energy in each pixel. Details on the chip design and measurements of the detector performance can be found in [4], [17], [18].

In contrast to its predecessor, the Timepix 3 has a data driven readout, which can handle hit rates up to 40 Mhits $\mathrm{cm}^{-2} \mathrm{~s}^{-1}$. The readout time per pixel (dead time), during which no other hit can be recognized, is around $475 \mathrm{~ns}$.

Energy depositions in the silicon sensor layer lead to the creation of electron-hole pairs. These charge carriers drift through the sensor layer due to an applied potential difference of $90 \mathrm{~V}$ between the common anode and the pixelated cathode. During their drift and collection the charge carriers induce a current at the pixel electrodes, which is shaped and amplified. In each pixel cell, the resulting voltage pulse is compared to an adjustable threshold level, corresponding to the minimum energy deposition required to trigger the pixel. This threshold level was set to $5 \mathrm{keV}$ for the presented experiment. As the pulse crosses this threshold a counter is started, which is stopped by the same pulse crossing the threshold level on its downward slope. The time interval the voltage pulse stays above the threshold level, the so-called Time-over-Threshold (ToT) is measured. This time interval was calibrated to ionizing energy depositions in the silicon by using the photo-electron signal after the illumination with X-rays of known energy [19]. With a counter of $40 \mathrm{MHz}$ clock frequency, energy resolutions of about $4 \mathrm{keV}$ could be achieved for the photo peak of a ${ }^{241} \mathrm{Am}$-source at $59.5 \mathrm{keV}$ [18].

In making measurements with the Timepix3 we have utilized beam triggers. When the first proton bunch of a macro-pulse strikes the tungsten target a trigger is generated. Upon receiving the trigger the time is set at zero and then the time after trigger is recorded for each interaction that follows. This continues until the first proton bunch from the subsequent macro-pulse restarts the timing sequence. To improve time resolution, two counters with different clock frequencies are integrated in the pixel electronics: A $40 \mathrm{MHz}$ counter is permanently running, providing the "slow" time-of-arrival value (sToA) of the interaction. Additionally, a $640 \mathrm{MHz}$ counter samples the time from the interaction until the next rising edge of the sToA-counter, giving time resolution of $1.56 \mathrm{~ns}$.

Due to the charge sharing effect caused by diffusion and repulsion of the charge carriers during their drift and due to the extended particle range, one particle interaction can lead to a charge deposition in several (adjacent) pixels, so that clusters in the pixel screen (tracks) are seen. Depending on particle type and energy depositions, different track shapes can be categorized [20]. Fig. 1 illustrates the recorded events in a time slice of $1 \mathrm{~ms}$ in the investigated neutron field.
To reduce overlap $100 \mu$ s long time-slices were chosen, during which individual pixel hits are filled into a frame (picture) and clusters were searched for, identified and categorized by geometric pattern recognition. Overlapping clusters were omitted by excluding events where at least one pixel was hit twice within the same time-slice. The same was done for clusters where the maximal time difference throughout the cluster exceeded $100 \mathrm{~ns}$. For each cluster the timestamp $t_{\text {cluster }}$ was defined as the first pixel to respond, i.e. the pixel with the lowest timestamp within the cluster. The energy deposited by the interaction, corresponding to the energy dissipated by ionization $E_{\text {ion }}$, is given by the sum of the energy measured by all pixels of one cluster.

\section{Time-of-Flight technique}

The number of registered clusters at a certain time after the reset due to the trigger signal is shown in Fig. 2. The obtained distribution represents the time structure of the micro-pulses: The $\gamma$-flashes of the spallation reaction are followed by the broad spectrum of neutrons. We measure the time between two consecutive $\gamma$-flashes with high precision to: $\Delta t_{\mu \mathrm{p}}=(1.78871 \pm 0.00002) \mu \mathrm{s}$.

The methodology developed for ToF measurements with Timepix devices by Bergmann et al. [20] was used. The timestamps were aligned to the $\gamma$-flashes that arrive at the site of detector $t_{\gamma}=\frac{d}{c}=71 \mathrm{~ns}$ after the spallation reaction, where $d=21.3 \mathrm{~m}$ is the distance from the detector to the tungsten target.

For interactions within the $i$-th micro-pulse $\left(t_{i-1}^{\gamma \text {-flash }}<\right.$ $t_{\text {cluster }}<t_{i}^{\gamma \text {-flash }}$ ) the neutron ToF is given by:

$$
t_{\mathrm{n}, \text { flight }}=t_{\text {cluster }}-t_{i}^{\gamma-\text { flash }}+\frac{d}{c} .
$$

This time can then be used to determine the kinetic energy of the impinging neutrons using the laws of special relativity:

$$
T=E-M=(\gamma-1) \times M,
$$

with

$$
\gamma=\left(1-\beta^{2}\right)^{-1 / 2}
$$

and

$$
\beta=\frac{t_{\gamma}}{t_{\mathrm{n}, \text { flight }}} .
$$

The measured number of events is presented as a function of neutron kinetic energy in Fig. 3 . Events caused by neutrons with energies up to $4 \mathrm{MeV}$ are mainly related to elastic and inelastic neutron scattering. With the two peaks resembling the resonances of elastic neutron scattering at their proper energies, we confirm the reliability of the neutron energy assignment by the ToF-technique. Above $2.75 \mathrm{MeV}$ nuclear reactions become visible and enhance the count rate. Neutron interactions in silicon are discussed in greater detail in section IV-A

A detailed study of the neutron interactions and their signatures as seen by a hybrid pixel detector of the Timepix family can be found in [20]. In this work the interactions 

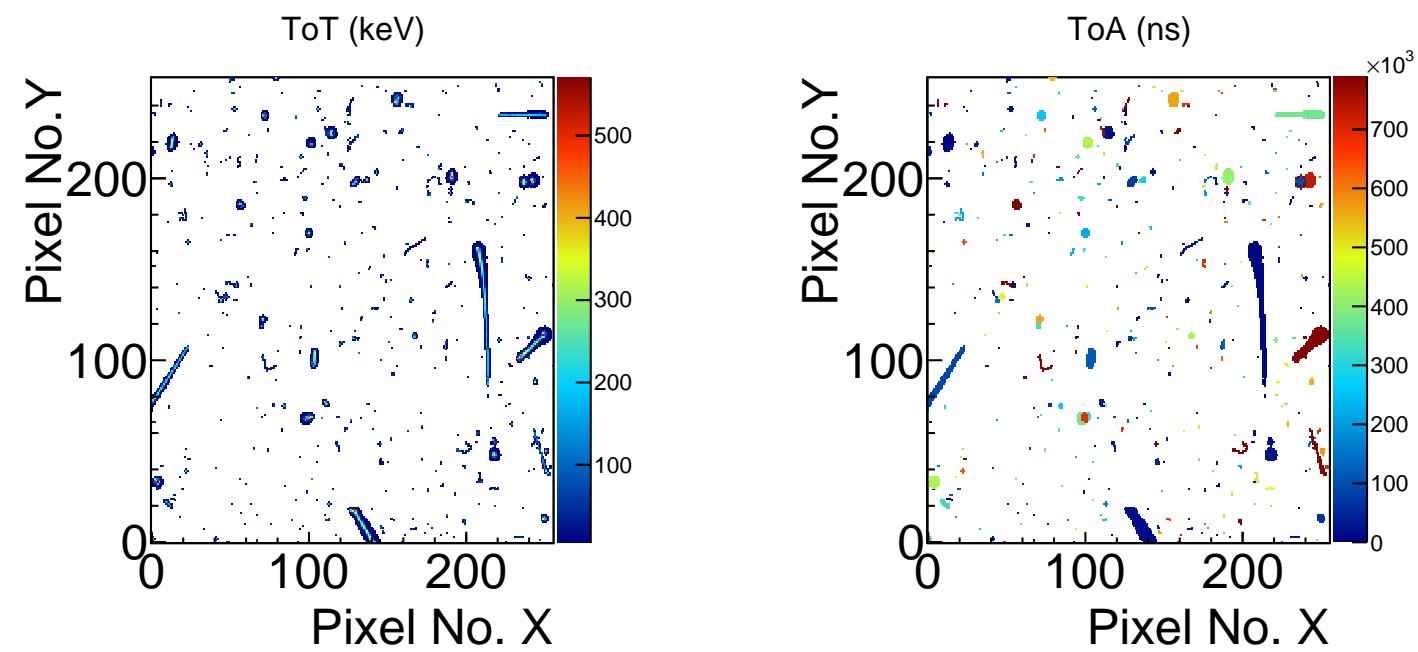

Fig. 1. Illustration of events measured within an integration time of $1 \mathrm{~ms}$. Different track shapes are seen, which can be used to characterize the radiation environment. The Timepix 3 allows the simultaneous measurement of the energy deposition (left screen) and the arrival time (right screen) of an interaction in the sensor layer.

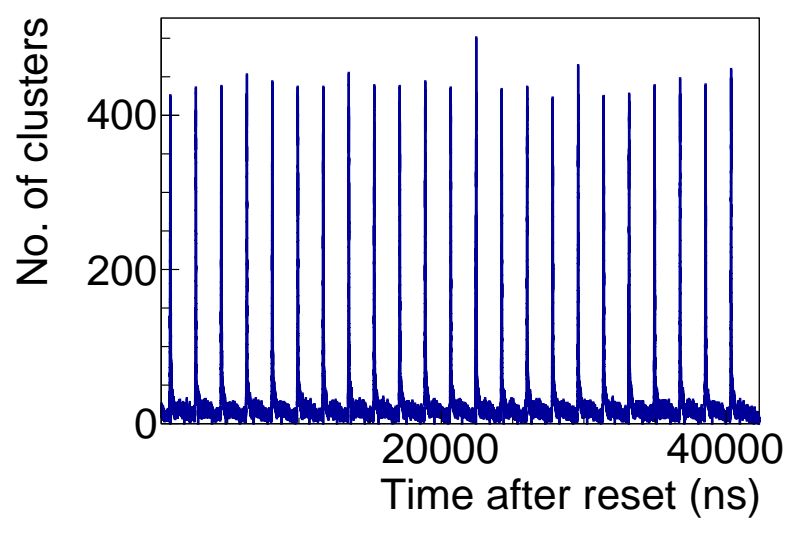

Fig. 2. Excerpt of the measured time spectrum. The sharp peaks due to the gamma flash are followed by the broad spectrum of neutrons.

are differentiated and discussed purely by means of energy depositions.

ToF ranges, corresponding to incident neutron energies, were selected to study the spectrum of ionizing energy depositions $E_{\text {ion }}$ to (quasi-)monochromatic neutron impact. A ToF interval length of $25 \mathrm{~ns}$ was chosen to account for the inaccuracy in the determination of the beginning of the micropulses and the jitter of the trigger (up to $10 \mathrm{~ns}$ ). With the detector's time resolution of $1.56 \mathrm{~ns}$ per bin, this interval corresponds to 16 energy bins. The mean energy

$$
\left\langle T_{\mathrm{n}}\right\rangle_{\mathrm{i}}=\frac{\int_{t}^{t+25 \mathrm{~ns}} N\left(t_{\text {cluster }}\right) \times T\left(t_{\text {cluster }}\right) d t_{\text {cluster }}}{\int_{t}^{t+25 \mathrm{~ns}} N\left(t_{\text {cluster }}\right) d t_{\text {cluster }}}
$$

was assigned to the energy spectrum of the $i$-th time interval,

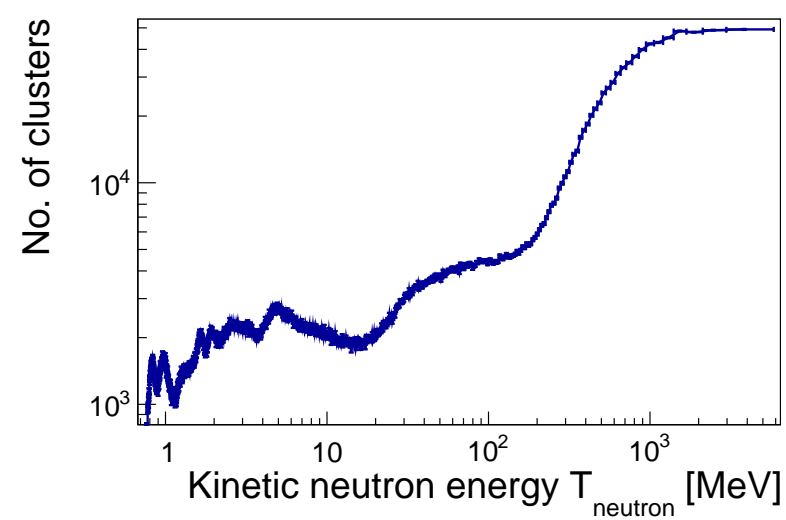

Fig. 3. Number of detected neutron interactions in the $300 \mu \mathrm{m}$ thick silicon sensor as a function of neutron kinetic energy. The neutron energies were assigned with the ToF technique (see text for explanation).

where the standard deviation

$$
\Delta T_{\mathrm{n}, i}=\sqrt{\frac{\int_{t}^{t+25 \mathrm{~ns}} N\left(t_{\text {cluster }}\right)\left[T\left(t_{\text {cluster }}\right)-\left\langle T_{\mathrm{n}}\right\rangle_{i}\right]^{2} d t_{\text {cluster }}}{\int_{t}^{t+25 \mathrm{~ns}} N\left(t_{\text {cluster }}\right) d t_{\text {cluster }}}}
$$

described its energy spread. Here, $N\left(t_{\text {cluster }}\right)$ is the number and $T\left(t_{\text {cluster }}\right)$ the kinetic energy of events registered with a timestamp $t_{\text {cluster. }}$. The relative energy resolution of each interval is shown as a function of neutron kinetic energy in Fig. 4

By employing the last micro-pulse in the series of macropulses the investigated energy range was extended to even lower energies than given by the separation of the micropulses. However, to accumulate sufficient statistics for the spectrum analysis, broader time bins had to be chosen. Still, energy resolutions $\frac{\Delta T_{n, i}}{\left\langle T_{n}\right\rangle_{i}}<3 \%$ could be achieved for incident 


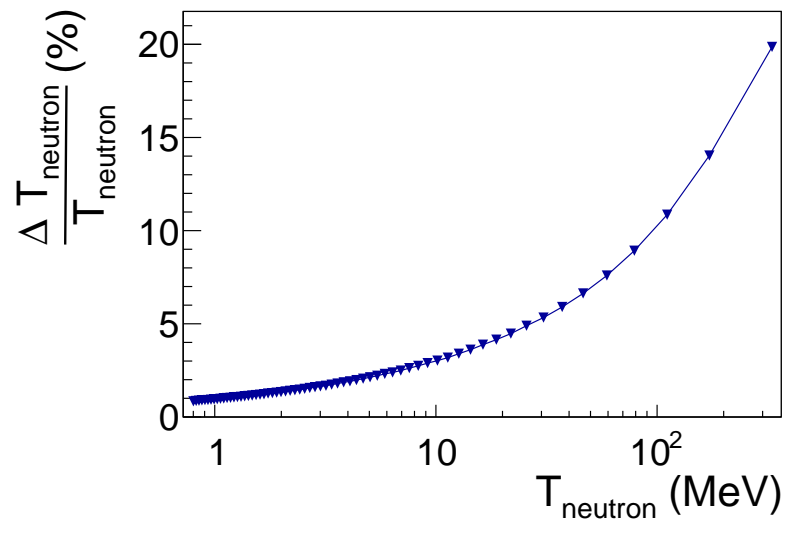

Fig. 4. Relative uncertainty of the neutron kinetic energy determination as a function of neutron kinetic energy.

neutron energies below $700 \mathrm{keV}$.

\section{RESUlts}

\section{A. Ionizing energy deposition spectra}

In Figs. 5,8 exemplary energy spectra of four selected energy intervals are presented. These spectra were interpreted based on the analysis of possible interactions of neutrons with ${ }^{28} \mathrm{Si}$ in the respective energy ranges. Due to the low abundance of ${ }^{29} \mathrm{Si}$ and ${ }^{30} \mathrm{Si}$, interactions of neutrons with these isotopes are neglected and not discussed in the following.

Neutrons with energies below $1.85 \mathrm{MeV}$ interact in silicon almost exclusively by elastic scattering: $\mathrm{Si}(\mathrm{n}, \mathrm{n}) \mathrm{Si}$. The energy transfer to the recoil silicon for incident neutron energy $T_{\mathrm{n}}$ is obtained from classical scattering theory by:

$$
T_{\mathrm{Si}}=\frac{2 M_{\mathrm{Si}} M_{\mathrm{n}}}{\left(M_{\mathrm{Si}}+M_{\mathrm{n}}\right)^{2}}(1-\cos \theta) \times T_{\mathrm{n}},
$$

where $\theta$ is the scattering angle in the center of mass system. The maximal energy transfer to the silicon for backscattered neutrons $(\theta=\pi)$ is:

$$
T_{\mathrm{Si}, \max }=\frac{4 M_{\mathrm{Si}} M_{\mathrm{n}}}{\left(M_{\mathrm{Si}}+M_{\mathrm{n}}\right)^{2}} \times T_{\mathrm{n}}=0.133 \times T_{\mathrm{n}} .
$$

On its path through the silicon sensor a recoil atom dissipates its energy by ionizing and non-ionizing energy losses. The ionizing energy losses at the maximum recoil energy are seen as edges in the energy deposition spectra. These edges are smeared out by the limited energy resolution of the detectors and the uncertainty of the incident neutron energy assignment. The energies at the edges $E_{\text {edge }}$ were obtained by fitting

$$
f(E)=\frac{1}{\exp \left(\frac{E-E_{\text {edge }}}{A}\right)+1}+B \times E+C
$$

to the energy spectra. The steepness of the edge is given by the parameter $A$. A linear background with slope $B$ and y-axis intercept $C$ was added to improve the fit quality and stability. The fit results are shown by the red line in Figs. 5.7 ,

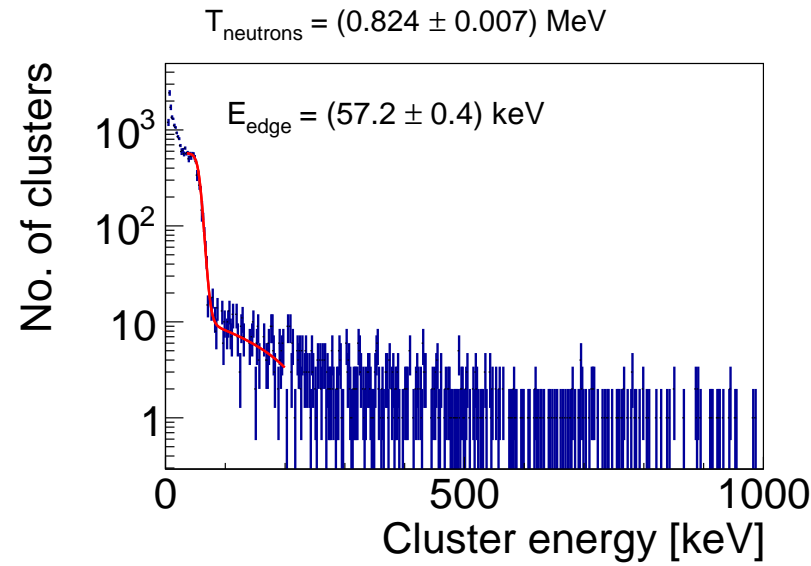

Fig. 5. Example of the deposited energy spectrum for neutron incident energy $T_{\mathrm{n}}=(0.824 \pm 0.007) \mathrm{MeV}$. The edge in the spectrum corresponds to the ionizing energy losses of recoil silicons created in neutron backscattering. The fit determining the energy value at the edge is shown by the red line.

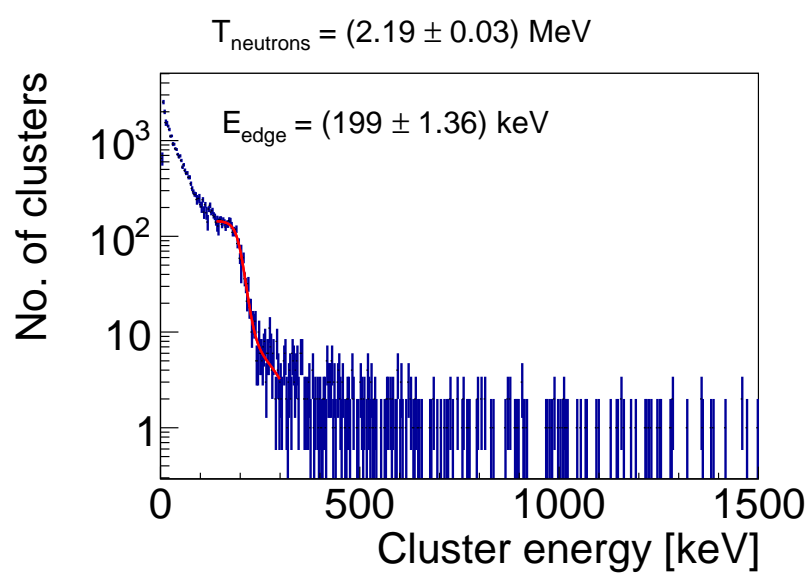

Fig. 6. Same as Fig. 5 but for neutron incident energy $T_{\mathrm{n}}=(2.19 \pm$ $0.03) \mathrm{MeV}$.

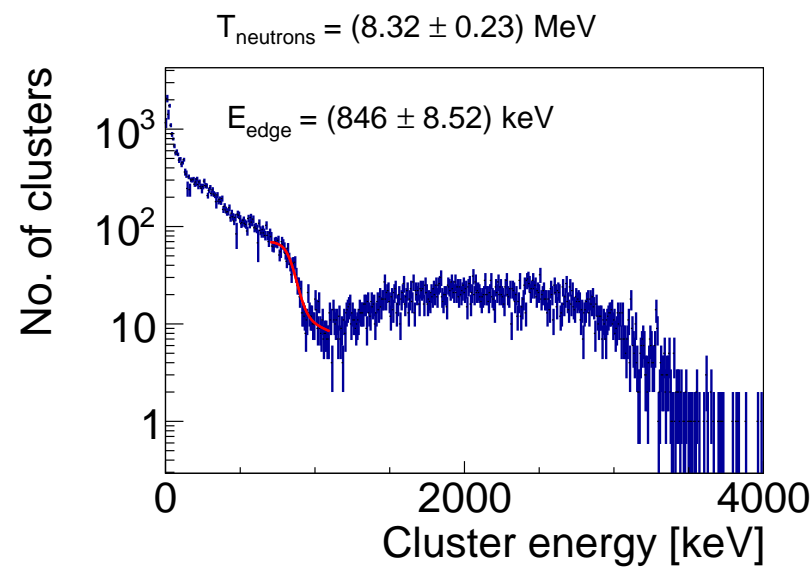

Fig. 7. Same as Fig. 5 but for neutron incident energy $T_{\mathrm{n}}=(8.32 \pm$ $0.23) \mathrm{MeV}$. 


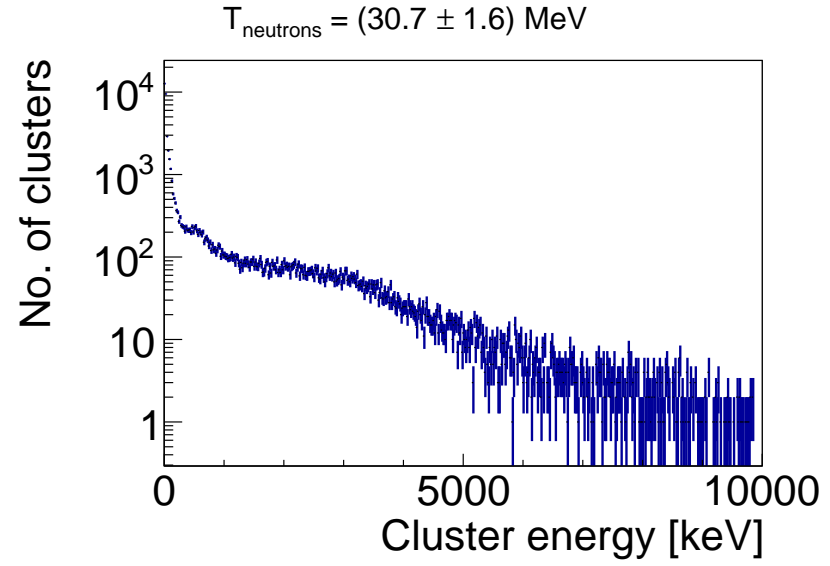

Fig. 8. Example of the measured energy deposition spectrum for neutron incident energy $T_{\mathrm{n}}=(30.7 \pm 1.6) \mathrm{MeV}$.

In Fig. 5 the energy spectrum for incident neutron energy $T_{\mathrm{n}}=(0.824 \pm 0.007) \mathrm{MeV}$ is presented. The fit finds the edge at $E_{\text {edge }}=(57.2 \pm 0.4) \mathrm{keV}$. This energy corresponds to the ionization produced by silicon recoil atoms from neutron backscattering of energy $T_{\mathrm{Si}, \max }=(109.6 \pm 0.9) \mathrm{keV}$ (calculated according to equation 14). The tail to higher energies is due to tracks from natural radioactive background and gamma rays or electrons from activation in the surroundings of the detector.

In Fig. 6 the energy spectrum for neutron impact energy $T_{\mathrm{n}}=(2.19 \pm 0.03) \mathrm{MeV}$ is shown. The edge of ionizing energy losses from elastic backscattering can be found at $E_{\text {edge }}=$ $(199.0 \pm 1.4) \mathrm{keV}$. The corresponding silicon recoil energy is $T_{\mathrm{Si}, \max }=(291.3 \pm 4.0) \mathrm{keV}$.

Above $1.85 \mathrm{MeV}$ inelastically scattered neutrons can excite the silicon nucleus to the first level: $\mathrm{Si}(\mathrm{n}, \mathrm{n}$ ')Si. The de-excitation of this state is accompanied by the emission of a gamma ray with energy $1.75 \mathrm{MeV}$. Due to their high energies, these gammas rarely interact in the silicon sensor layer so that their contribution to the energy deposition spectra is negligible. However, in the same way as in neutron elastic scattering the recoil silicon loses part of its energy by a detectable ionizing signal. Given the large separation of the first level from the ground state, the edge of the recoil silicon energy depositions from neutron inelastic backscattering is at significantly lower energies than the one of recoil silicon from elastic backscattering.

In addition to the processes discussed above, in Fig. 7 a broad continuum of energies up to approximately $4 \mathrm{MeV}$ for neutron impact energy $T_{\mathrm{n}}=(8.32 \pm 0.23) \mathrm{MeV}$ can be seen. This is due to the energy depositions of the charged products from the nuclear ${ }^{28} \mathrm{Si}(\mathrm{n}, \mathrm{X})$-reactions:

$$
\begin{aligned}
{ }^{28} \mathrm{Si}+n & \rightarrow{ }^{25} \mathrm{Mg}+\alpha, Q=-2.75 \mathrm{MeV} \\
& \rightarrow{ }^{28} \mathrm{Al}+\mathrm{p}, \quad Q=-4 \mathrm{MeV}
\end{aligned}
$$

The products of the reactions take away the available energy as kinetic energy.
As the interaction products are produced at the same time and in the same place, they are detected as one single cluster, with a cluster energy given by the sum of the products' energy depositions. Due to the saturation of the Timepix electronics [21] and recombination of charge carriers, for high local charge depositions in the sensor layer the energy content of the cluster is underestimated, so that we find the maximal energy depositions at approximately $4 \mathrm{MeV}$ in the spectrum.

Still, on top of this broad continuum, the edge (at $\left.E_{\text {edge }}=(846 \pm 9) \mathrm{keV}\right)$ due to the ionizing energy depositions of the recoil silicon $\left(T_{\mathrm{Si}, \max }=(1,107 \pm 31) \mathrm{keV}\right)$ can be fitted.

At higher neutron energies (see the spectrum for $T_{\mathrm{n}}=(30.7 \pm 1.6) \mathrm{MeV}$ in Fig. 8) the interpretation of the spectra is more complex, as the number of possible interaction channels increases. Moreover, silicon recoil atoms and the products of nuclear reactions deposit high enough energy to saturate the pixel electronics and to enhance the recombination of charge carriers. The presented energy deposition spectrum is thus a superposition of energy depositions by the products of the reaction channels, convoluted with the detector responses. Edges of energy depositions by different processes are smeared out and overlap, resulting in a broad continuum.

\section{B. Ionizing energy depositions of recoil silicon in the silicon lattice}

From the ionizing energy depositions as fitted in section IV-A the fraction $f_{\text {ion }}$ of the ionizing energy loss at the recoil energy $T_{\mathrm{Si} \text { max }}$ is given by

$$
f_{\text {ion }}=\frac{E_{\text {edge }}}{T_{\text {Si,max }}} \overbrace{=}^{\text {Eq. 14 }} \frac{E_{\text {edge }}}{0.133 \times T_{\mathrm{n}}},
$$

with the error given according to the Gaussian error propagation as:

$$
\Delta f_{\text {ion }}=f_{\text {ion }} \times \sqrt{\left(\frac{\Delta E_{\text {edge }}}{E_{\text {edge }}}\right)^{2}+\left(\frac{\Delta T_{\mathrm{n}}}{T_{\mathrm{n}}}\right)^{2}} .
$$

For the recoil silicon energy range up to $800 \mathrm{keV}, \Delta E_{\text {edge }}$ was determined by the fit errors. Above, to account for the uncertainty due to charge recombination and the saturation of the pixel electronics an additional error of $10 \%$ of the recoil energy was introduced.

In Fig. 9 the ionizing energy losses $f_{\text {ion }}$ are shown as a function of the silicon recoil energy. For completeness, the neutron kinetic energies leading to recoils of the given energies are indicated.

The results of the presented measurement are compared with the theoretical prediction of Norgett, Torrens and Robinson (equation (5) with the unviversal funcion of equation (3), black solid line), the model of Akkerman (equation (5) with the universal function of equation (6), yellow solid line) and the data of Sattler [6] (red points). Using the ToF technique, the significance compared to the previous measurement was improved by extending the amount of data points and, for 


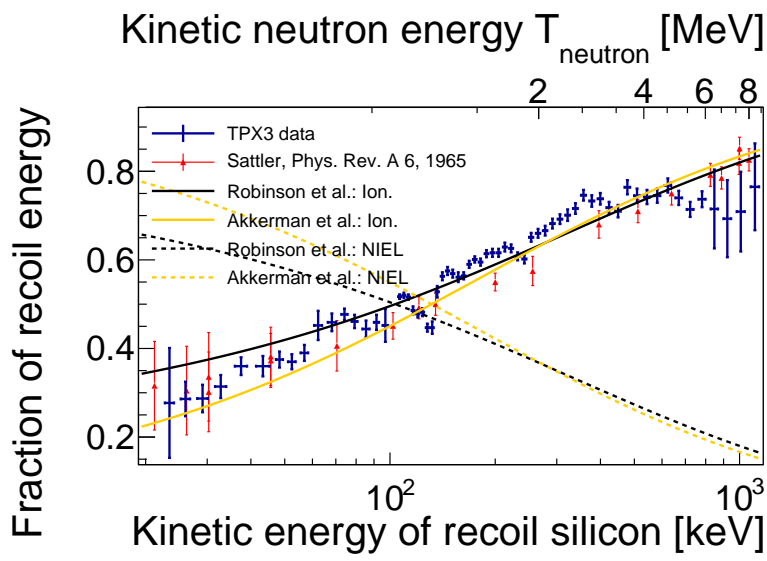

Fig. 9. Fraction of the (inelastic) ionizing energy losses of a silicon recoil atom as a function of its energy. The data measured with the Timepix 3 are compared with the data from Sattler (red points) and the partition functions according to Norgett, Torrens and Robinson (black solid line) and Akkerman and Barak (yellow solid line). The incident fast neutron energies producing the energetic silicon recoil atoms and the calculated fraction of the NIEL for both models are indicated for completeness.

recoil silicon energies below $100 \mathrm{keV}$, by a better energy resolution of the incident neutron energy. The presented data confirm the trend of the previous measurement to be closer to the predicted partition function of Akkerman and Barak than to the one of Norgett, Torrens and Robinson. However, with the measurement uncertainties, the latter prediction cannot be ruled out.

\section{CONCLUSION}

The energy depositions in a silicon sensor layer of $300 \mu \mathrm{m}$ thickness after fast neutron impact in the range from hundreds of $\mathrm{keV}$ up to hundreds of $\mathrm{MeV}$ were studied. Neutron energies were assigned by using the ToF technique.

Measured ionizing energy deposition spectra were presented and interpreted. By spectrum analysis the competition of the ionizing energy losses versus the non-ionizing energy losses of silicon recoils (primary-knock on atoms) in the silicon lattice was studied. Being consistent with the previous measurement of Sattler, the results show a closer agreement with the partition function of Akkerman and Barak than with the one of Norgett, Torrens and Robinson.

\section{ACKNOWLEDGMENT}

We acknowledge the expert help of Dr. S. Wender in assisting with the experimental setup and measurements. The work has been done in the frame of the Medipix collaboration. This research project has been supported by a Marie Curie Early Initial Training Network Fellowship of the European Community's Seventh Framework Programme under contract number (PITN-GA-2011-289198-ARDENT), the Ministry of Education, Youth and Sports of the Czech Republic under project number LM2015058 and in part by the United States Department of Energy Contract No. DE-SC0012704. We have benefited from the use of the Los Alamos Neutron Science Center at LANL, which is funded by the U.S. Department of Energy under Contract No. DE-AC52-06NA25396.

\section{REFERENCES}

[1] G. Lindström, M. Moll, and E. Fretwurst, "Radiation hardness of silicon detectors - a challenge from high-energy physics," Nuclear Instruments and Methods in Physics Research Section A: Accelerators, Spectrometers, Detectors and Associated Equipment, vol. 426, no. 1, pp. 1 - 15, 1999. [Online]. Available: http: //www.sciencedirect.com/science/article/pii/S0168900298014624

[2] C. Leroy and P.-G. Rancoita, "Particle interaction and displacement damage in silicon devices operated in radiation environments," Reports on Progress in Physics, vol. 70, no. 4, p. 493, 2007. [Online]. Available: http://stacks.iop.org/0034-4885/70/i=4/a=R01

[3] Y. Tosaka, H. Kanata, T. Itakura, and S. Satoh, "Simulation technologies for cosmic ray neutron-induced soft errors: Models and simulation systems," IEEE Transaction on Nuclear Science, vol. 46, June 1999.

[4] T. Poikela, J. Plosila, T. Westerlund, M. Campbell, M. D. Gaspari, X. Llopart, V. Gromov, R. Kluit, M. van Beuzekom, F. Zappon, V. Zivkovic, C. Brezina, K. Desch, Y. Fu, and A. Kruth, "Timepix3: a $65 \mathrm{k}$ channel hybrid pixel readout chip with simultaneous toa/tot and sparse readout," Journal of Instrumentation, vol. 9, no. 05, p. C05013, 2014. [Online]. Available: http://stacks.iop.org/1748-0221/9/i= $05 / \mathrm{a}=\mathrm{C} 05013$

[5] J. Lindhard, V. Nielsen, M. Scharff, and P. Thomsen, Integral equations governing radiation effects. Munksgaard i komm., 1963.

[6] A. R. Sattler, "Ionization produced by energetic silicon atoms within a silicon lattice," Phys. Rev., vol. 138, pp. A1815-A1821, Jun 1965. [Online]. Available: http://link.aps.org/doi/10.1103/PhysRev.138.A1815

[7] G. H. Kinchin and R. S. Pease, "The displacement of atoms in solids by radiation," Reports on Progress in Physics, vol. 18, no. 1, p. 1, 1955. [Online]. Available: http://stacks.iop.org/0034-4885/18/i=1/a=301

[8] M. Norgett, M. Robinson, and I. Torrens, "A proposed method of calculating displacement dose rates," Nuclear Engineering and Design, vol. 33, no. 1, pp. 50 - 54, 1975. [Online]. Available: http://www.sciencedirect.com/science/article/pii/0029549375900357

[9] M. T. Robinson and I. M. Torrens, "Computer simulation of atomicdisplacement cascades in solids in the binary-collision approximation," Phys. Rev. B, vol. 9, pp. 5008-5024, Jun 1974. [Online]. Available: http://link.aps.org/doi/10.1103/PhysRevB.9.5008

[10] M. T. Robinson, "Basic physics of radiation damage production," Jour nal of Nuclear Materials, vol. 216, pp. 1 - 28, 1994. [Online]. Available: http://www.sciencedirect.com/science/article/pii/0022311594900035

[11] - "Energy dependence of neutron irradiation damage in solids," British Nuclear Energy Society. Nuclear Fusion Reactors, September 1969, p. 364.

[12] A. Akkerman and J. Barak, "New partition factor calculations for evaluating the damage of low energy ions in silicon," IEEE Transactions on Nuclear Science, vol. 53, no. 6, pp. 3667-3674, Dec 2006.

[13] I. Jun, W. Kim, and R. Evans, "Electron nonionizing energy loss for device applications," IEEE Transactions on Nuclear Science, vol. 56, no. 6, pp. 3229-3235, Dec 2009.

[14] P. W. Lisowski and K. F. Schoenberg, "The los alamos neutron science center," Nuclear Instruments and Methods in Physics Research Section A: Accelerators, Spectrometers, Detectors and Associated Equipment, vol. 562, no. 2, pp. $910-914,2006$, proceedings of the 7th International Conference on Accelerator ApplicationsAccApp057th International Conference on Accelerator Applications. [Online]. Available: http: //www.sciencedirect.com/science/article/pii/S0168900206003792

[15] "Medipix3 collaboration." [Online]. Available: http://www.cern.ch/ medipix

[16] X. Llopart, R. Ballabriga, M. Campbell, L. Tlustos, and W. Wong, "Timepix, a $65 \mathrm{k}$ programmable pixel readout chip for arrival time, energy and/or photon counting measurements," Nuclear Instruments and Methods in Physics Research Section A: Accelerators, Spectrometers, Detectors and Associated Equipment, vol. 581, no. 1-2, pp. 485 - 494, 2007, \{VCI $\}$ 2007Proceedings of the 11th International Vienna Conference on Instrumentation. [Online]. Available: http: //www.sciencedirect.com/science/article/pii/S0168900207017020

[17] M. D. Gaspari, J. Alozy, R. Ballabriga, M. Campbell, E. Fröjdh, J. Idarraga, S. Kulis, X. Llopart, T. Poikela, P. Valerio, and W. Wong, "Design of the analog front-end for the timepix3 and smallpix hybrid pixel detectors in $130 \mathrm{~nm}$ cmos technology," Journal of Instrumentation, vol. 9, no. 01, p. C01037, 2014. [Online]. Available: http://stacks.iop.org/1748-0221/9/i=01/a=C01037

[18] E. Frojdh, M. Campbell, M. D. Gaspari, S. Kulis, X. Llopart, T. Poikela, and L. Tlustos, "Timepix3: first measurements and characterization of a hybrid-pixel detector working in event driven mode," Journal of 
Instrumentation, vol. 10, no. 01, p. C01039, 2015. [Online]. Available: http://stacks.iop.org/1748-0221/10/i=01/a=C01039

[19] J. Jakubek, "Precise energy calibration of pixel detector working in time-over-threshold mode," Nuclear Instruments and Methods in Physics Research Section A: Accelerators, Spectrometers, Detectors and Associated Equipment, vol. 633, Supplement 1, pp. S262 - S266, 2011, 11th International Workshop on Radiation Imaging Detectors (IWORID). [Online]. Available: http://www.sciencedirect.com/science/ article/pii/S0168900210013732

[20] B. Bergmann, R. O. Nelson, J. M. O'Donnell, S. Pospisil, J. Solc, H. Takai, and Z. Vykydal, "Time-of-flight measurement of fast neutrons with timepix detectors," Journal of Instrumentation, vol. 9, no. 05, p. C05048, 2014. [Online]. Available: http: //stacks.iop.org/1748-0221/9/i=05/a=C05048

[21] C. Granja, J. Jakubek, U. Köster, M. Platkevic, and S. Pospisil, "Response of the pixel detector timepix to heavy ions," Nuclear Instruments and Methods in Physics Research Section A: Accelerators, Spectrometers, Detectors and Associated Equipment, vol. 633 , Supplement 1, pp. S198 - S202, 2011, 11th International Workshop on Radiation Imaging Detectors (IWORID). [Online]. Available: http://www.sciencedirect.com/science/article/pii/S0168900210013562 